\title{
HIGHER CONJUGATION COHOMOLOGY IN COMMUTATIVE HOPF ALGEBRAS
}

\author{
M. D. CROSSLEY ${ }^{1}$ AND SARAH WHITEHOUSE ${ }^{2}$ \\ ${ }^{1}$ Climatic Research Unit, University of East Anglia, \\ Norwich NR4 7TJ, UK (crossley@member.ams.org) \\ ${ }^{2}$ Laboratoire de Géométrie-Algèbre, Université d'Artois-Pôle de Lens, \\ Rue Jean Souvraz, S.P. 18-63207 Lens, France \\ (whitehouse@euler.univ-artois.fr)
}

(Received 29 July 1999)

\begin{abstract}
Let $A$ be a graded, commutative Hopf algebra. We study an action of the symmetric group $\Sigma_{n}$ on the tensor product of $n-1$ copies of $A$; this action was introduced by the second author in [8] and is relevant to the study of commutativity conditions on ring spectra in stable homotopy theory [6].

We show that for a certain class of Hopf algebras the cohomology ring $H^{*}\left(\Sigma_{n} ; A^{\otimes n-1}\right)$ is independent of the coproduct provided $n$ and $(n-2)$ ! are invertible in the ground ring. With the simplest coproduct structure, the group action becomes particularly tractable and we discuss the implications this has for computations.
\end{abstract}

Keywords: Hopf algebras; cohomology operations; cohomology of groups; representations of symmetric groups

AMS 2000 Mathematics subject classification: Primary 16W30; 57T05; 20C30; 20J06; 55S25

\section{Introduction and main results}

Let $A$ be a graded, connected, unital, counital, associative, coassociative Hopf algebra. In $\S 8$ of [5] it was shown how $A$ has a 'conjugation' or 'antipode' $\chi$ satisfying the equality

$$
\mu \circ(1 \otimes \chi) \circ \Delta=\eta \circ \epsilon,
$$

where $\mu$ and $\Delta$ are the product and coproduct and $\eta$ and $\epsilon$ are the unit and counit. In particular, $\chi(1)=1$ and, for $x$ of positive degree,

$$
\chi(x)=-x+\sum x^{\prime} \chi\left(x^{\prime \prime}\right),
$$

where $\Delta(x)=x \otimes 1+1 \otimes x+\sum x^{\prime} \otimes x^{\prime \prime}$. If $A$ is commutative, then $\chi^{2}=1$ and so gives an action of $\Sigma_{2}$ on $A$.

The second author extended this in [8] by providing, for each $n>2$, an action of the symmetric group $\Sigma_{n}$ on $A^{\otimes n-1}$ when $A$ is commutative. 
If $\sigma_{i}$ denotes the transposition $i \leftrightarrow i+1$, then $\Sigma_{n}$ is generated by $\sigma_{1}, \ldots, \sigma_{n-1}$, and the action on $A^{\otimes n-1}$ is given by:

$$
\begin{aligned}
\sigma_{1} & =[(\mu \otimes 1) \circ(\chi \otimes \Delta)] \otimes 1^{\otimes n-3}, \\
\sigma_{i} & =1^{\otimes i-2} \otimes[(1 \otimes(\mu \circ(\mu \otimes 1)) \otimes 1) \circ(\Delta \otimes \chi \otimes \Delta)] \otimes 1^{\otimes n-i-2} \quad \text { if } 1<i<n-1, \\
\sigma_{n-1} & =1^{\otimes n-3} \otimes[(1 \otimes \mu) \circ(\Delta \otimes \chi)] .
\end{aligned}
$$

Note that each $\sigma_{i}$ acts multiplicatively, as does $\chi$ in the case where $n=2$.

Thus if $A$ is commutative, as we will henceforth assume, we have a multiplicative action of $\Sigma_{n}$ on $A^{\otimes n-1}$ for each $n \geqslant 2$. While it may seem unusual to have $\Sigma_{n}$ acting on an $n-1$-fold product, this action does arise quite naturally in stable homotopy theory. If $E$ is a sufficiently nice ring spectrum and $A=\pi_{*}(E \wedge E)$, the set of 'cooperations' in the cohomology theory associated to $E$, then $A$ naturally has the structure of a commutative Hopf algebra (or, more generally, that of a Hopf algebroid). The map induced on $A$ by switching the factors in the smash product $E \wedge E$ is precisely the conjugation and this generalizes to give the 'higher conjugations' in the following way. We take $\pi_{*}(E \wedge \cdots \wedge E)$, the homotopy of a smash product of $n$ copies of $E$. This naturally has an action of $\Sigma_{n}$. But $\pi_{*}\left(E^{\wedge n}\right)=E_{*}\left(E^{\wedge n-1}\right)$, the $E$-cohomology of an $n-1$-fold product of copies of $E$. For suitable $E$ (examples are given in [1] ), this is isomorphic to $A^{\otimes n-1}$. The proof that this 'topological' action can be described algebraically and thus extended to all Hopf algebroids can be found in [8].

With a view to understanding commutativity in ring spectra, we would like to understand these higher conjugation actions. Suitable information would be the cohomology $H^{*}\left(\Sigma_{n} ; A^{\otimes n-1}\right)$ of $\Sigma_{n}$ with coefficients in $A^{\otimes n-1}$. For example, we need to know the $\Sigma_{n}$-invariants, since these form $H^{0}$. However, in [3] we saw how complicated this calculation could be when we attempted it for $n=2$ and for $A$ an object familiar to algebraic topologists: the mod 2 dual Steenrod algebra.

Since these $\Sigma_{n}$ actions explicitly involve $\chi$ and $\Delta$, and the former involves the latter, it would be desirable if we could make the coproduct $\Delta$ as simple as possible. The following theorem, which is our main result and is proved in $\S 2$, gives conditions under which we can do this without changing the cohomology ring that we wish to calculate.

Theorem 1.1. Let $A$ be a graded, connected, coassociative Hopf algebra that is free as a module over the ring $R$. Suppose that $A$ is isomorphic, as an algebra, to a tensor product of associative Hopf algebras, each of which has just a single algebra generator. Let $\tilde{A}$ be this tensor product, considered as a Hopf algebra. If $n$ and $(n-2)$ ! are invertible in $R$ then there is an isomorphism of algebras

$$
\tilde{A}^{\otimes n-1} \longrightarrow A^{\otimes n-1},
$$

which commutes with the $\Sigma_{n}$ action, thus inducing an isomorphism of cohomology rings

$$
H^{*}\left(\Sigma_{n} ; \tilde{A}^{\otimes n-1}\right) \approx H^{*}\left(\Sigma_{n} ; A^{\otimes n-1}\right) .
$$

In particular, if $A$ is a graded, connected, commutative, biassociative Hopf algebra of finite type and $R$ is a perfect field, then, by the Borel-Hopf Theorem [5, Theorem 7.11], 
$A$ satisfies the hypotheses of Theorem 1.1. Our theorem is more general than this in that we can work over a ring instead of a field, and we do not need $A$ to be of finite type, so, for example, $A=\mathbb{Z} / 8\left[x_{1}, x_{2}, \ldots\right]$, where $\left|x_{i}\right|=2$ for all $i$, satisfies the hypotheses of Theorem 1.1, but not of the Borel-Hopf Theorem.

Note that by assuming that the underlying algebra of $A$ is a tensor product of associative monogenic algebras, we automatically have that $A$ is commutative (and associative).

The importance of Theorem 1.1 rests on the fact that the coproduct in $\tilde{A}$ will generally be much simpler than that in $A$, since all the generators of $\tilde{A}$ are primitive.

In particular, the action on $\tilde{A}^{\otimes n-1}$ is 'linear' in the sense that there is a subspace $V$, preserved by the group action, such that $\tilde{A}^{\otimes n-1}$ is the (truncated) polynomial algebra on $V$ and the action on $\tilde{A}^{\otimes n-1}$, being multiplicative, is determined by the action on $V$. Thus many results from classical invariant theory, e.g. Molien's Theorem on the Poincaré series for the invariants $[\mathbf{2}, \mathbf{7}]$, can be applied to $\tilde{A}^{\otimes n-1}$ and, via Theorem 1.1, to $A^{\otimes n-1}$. In fact one can go further, since the representation $V$ is not hard to analyse. It is a direct sum of copies of a certain $n$-1-dimensional representation $W$, with one copy of $W$ for each algebra generator of $A$ (with corresponding degree). We thank Ian Leary for the observation that $W$ can be identified with the familiar $n$-1-dimensional representation of $\Sigma_{n}$ constructed as follows. Consider an $n$-dimensional vector space with basis $e_{1}, \ldots, e_{n}$ permuted by $\Sigma_{n}$ and take the subspace spanned by $e_{1}-e_{2}, e_{2}-e_{3}, \ldots, e_{n-1}-e_{n}$, which is clearly preserved by the group action.

The hypothesis that $n(n-2)$ ! be invertible is rather curious. Some such condition is certainly required; [3] illustrates this in the case where $n=2$ and similar examples can be constructed for larger $n$ showing that $n$, at least, must be invertible. We know of no examples where $n$ is invertible and the conclusion of the theorem does not hold but the condition that $n(n-2)$ ! be invertible does occur naturally in a related setting. The representation $W$, mentioned above, can be obtained as $R \Sigma_{n} \cdot e$ for some idempotent $e \in R \Sigma_{n}$ if, and only if, $n(n-2)$ ! is invertible in $R$. (The value $n(n-2)$ ! arises here as the order of the group, $n$ !, divided by the dimension of $W, n-1$.) One such idempotent plays a central role in our proof of Theorem 1.1, which might suggest that its existence is tied to the validity of the theorem. However, it is equally possible that there is a more general proof of the theorem than that presented here.

If we make the stronger assumption that $n$ ! is invertible in $R$, then the cohomology ring is zero in positive degrees, but even in this case the theorem is of significance since it greatly simplifies the calculation of $H^{0}\left(\Sigma_{n} ; A^{\otimes n-1}\right)=\left(A^{\otimes n-1}\right)^{\Sigma_{n}}$.

Conversely, if we remove the assumption on $n(n-2)$ !, then we can no longer apply the theorem, but there is still a relation between $A^{\otimes n-1}$ and $\tilde{A}^{\otimes n-1}$. For example, if $R$ is a field then the $\Sigma_{n}$ invariants in the former can be injectively mapped into those of the latter. This follows from the fact that, filtering by powers of the augmentation ideal, the lowest filtration part of an invariant in $A^{\otimes n-1}$ is an invariant in $\tilde{A}^{\otimes n-1}$.

Finally, we end this section by observing how, in the case where $n=2$, Theorem 1.1 leads to the following 'model' for the conjugation invariants.

Theorem 1.2. Suppose that $A$ is as in Theorem 1.1 and that 2 is invertible in the ground ring. Let $A^{\Sigma_{2}}$ denote the subalgebra of conjugation invariants, and $A_{\mathrm{E}}$ the sub- 
algebra of $A$ spanned by the monomials whose exponents sum to an even number. Then there is an isomorphism of algebras

$$
A^{\Sigma_{2}} \approx A_{\mathrm{E}}
$$

This is a simple consequence of Theorem 1.1 and the fact that $\chi(x)=-x$ if $x$ is primitive.

We note that if $p>2$ then Theorem 1.2 satisfactorily solves the 'conjugation invariants' problem for the mod $p$ dual Steenrod algebra, in marked contrast to the partial solution [3] available when $p=2$.

\section{Proof of Theorem 1.1}

The proof is entirely constructive: we give an explicit definition of a map $f: \tilde{A}^{\otimes n-1} \rightarrow$ $A^{\otimes n-1}$ and proceed to show that it has the required properties. In order to state the definition of $f$, we need some notation. If $x$ is an element of $A$ then we write $x[i]$ for the element $1 \otimes \cdots \otimes 1 \otimes x \otimes 1 \otimes \cdots \otimes 1$ in $A^{\otimes n-1}$, where $x$ occurs in the $i$ th place. Similar notation is used for elements of $\tilde{A}^{\otimes n-1}$.

Let $f: \tilde{A}^{\otimes n-1} \rightarrow A^{\otimes n-1}$ be defined as follows. For every primitive element $\tilde{x}$ in $\tilde{A}$ we set

$$
f(\tilde{x}[i])=\left(\sigma_{i}-1\right)\left(\sigma_{i-1}-1\right) \cdots\left(\sigma_{1}-1\right) T_{n} x[1],
$$

where $T_{n} \in R \Sigma_{n}$ is the sum of all elements in the subgroup $G_{n}$ of $\Sigma_{n}$ generated by $\sigma_{2}, \ldots, \sigma_{n-1}$. We then extend $f$ multiplicatively, noting that, since $\tilde{A}$ (and, hence, $\tilde{A}^{\otimes n-1}$ ) is primitively generated, this defines $f$ on the whole algebra.* Before going any further we must verify that such a map is well defined, since $\tilde{A}^{\otimes n-1}$ is not necessarily free.

Lemma 2.1. The map $f$ is well defined.

Proof. Essentially, the conditions placed on the algebraic structure of $A$ (and hence that of $\tilde{A}$ ) in the theorem serve to ensure that $\tilde{A}^{\otimes n-1}$ is 'free enough' for the definition of $f$ to work. For we have stated that $A$ should be a tensor product of monogenic Hopf algebras and this is a strong restriction, as is discussed in $[\mathbf{4}, \S \S 1-3]$. Monogenic Hopf algebras that are free as $R$-modules must have the form $R[x]$ or $R[x] /\left(x^{h}\right)$, where $h$ is either 2 , if $x$ has odd degree and $R$ has characteristic different from 2 , or $p^{r}$ if $p$ is the characteristic of $R$. So then the only relations occurring in $\tilde{A}^{\otimes n-1}$ are of the form $\tilde{x}[i]^{h}=0$ for some $h$ dependent on $x$ (and not on $i$ ). Now $f\left(\tilde{x}[i]^{h}\right)$ can be written as $\left(\left(g_{1} \pm g_{2} \pm \cdots \pm g_{k}\right) x[1]\right)^{h}$ for some $g_{1}, \ldots, g_{k} \in \Sigma_{n}$. If $x$ has odd degree and $R$ has characteristic different from 2 (so that $h$ will be 2 ), then $\left(g_{1} \pm \cdots \pm g_{k}\right) x[1]$ will also have odd degree so its square will be zero, and $f$ preserves the relation $\tilde{x}[i]^{2}=0$. On the other hand, if $h=p^{r}$ then, since $R$ has characteristic $p$, the operation of taking $p^{r}$ th powers is linear, so $f\left(\tilde{x}[i]^{h}\right)=\left(g_{1} x[1]\right)^{h} \pm \cdots \pm\left(g_{k} x[1]\right)^{h}=g_{1}\left(x[1]^{h}\right) \pm \cdots \pm g_{k}\left(x[1]^{h}\right)$, since the group acts multiplicatively. Since the relations holding in $A^{\otimes n-1}$ are the same as those

\footnotetext{
* We note that $\left(\sigma_{1}-1\right) T_{n} \cdot\left(\sigma_{1}-1\right) T_{n}=n(n-2) !\left(\sigma_{1}-1\right) T_{n}$ in $R \Sigma_{n}$, so that $e=\left(\sigma_{1}-1\right) T_{n} / n(n-2)$ ! is an idempotent, and that $R \Sigma_{n} \cdot e$ is the $n-1$-dimensional representation $W$ mentioned in $\S 1$. This idempotent $e$ is intrinsic to $f$ in that $f$ is determined by $f(\tilde{x}[1])=\left(\sigma_{1}-1\right) T_{n} x[1]$.
} 
holding in $\tilde{A}^{\otimes n-1}$ (they are the same as algebras), we have $x[1]^{h}=0$ in $A^{\otimes n-1}$ and so $f$ also preserves the relation $\tilde{x}[i]^{h}=0$. This completes the proof that $f$ is well defined.

Lemma 2.2. The map $f$ commutes with the action of $\Sigma_{n}$.

Proof. Since both $f$ and the group elements act multiplicatively, it is sufficient to check this for a set of generators, and we will check it on the primitive elements of $\tilde{A}^{\otimes n-1}$. So throughout the proof we let $\tilde{x} \in \tilde{A}$ denote a primitive element. Then $\tilde{x}[i]$ is a primitive in $\tilde{A}^{\otimes n-1}$ and all primitives in that algebra occur in this way.

From the definition of the $\Sigma_{n}$ action we see that for $\tilde{x}$ primitive, the group elements $\sigma_{k}$ act as follows:

$$
\sigma_{k} \tilde{x}[i]= \begin{cases}\tilde{x}[i] & \text { if } k>i+1, \\ \tilde{x}[i]+\tilde{x}[i+1] & \text { if } k=i+1, \\ -\tilde{x}[i] & \text { if } k=i, \\ \tilde{x}[i-1]+\tilde{x}[i] & \text { if } k=i-1, \\ \tilde{x}[i] & \text { if } k<i-1,\end{cases}
$$

and so we must show

$$
\sigma_{k} f(\tilde{x}[i])= \begin{cases}f(\tilde{x}[i]) & \text { if } k>i+1, \\ f(\tilde{x}[i])+f(\tilde{x}[i+1]) & \text { if } k=i+1, \\ -f(\tilde{x}[i]) & \text { if } k=i, \\ f(\tilde{x}[i-1])+f(\tilde{x}[i]) & \text { if } k=i-1, \\ f(\tilde{x}[i]) & \text { if } k<i-1,\end{cases}
$$

and we will prove this case by case.

If $k>i+1$ then $\sigma_{k} f(\tilde{x}[i])=f(\tilde{x}[i])$, since $\sigma_{k}$ commutes with $\left(\sigma_{i}-1\right) \cdots\left(\sigma_{1}-1\right)$, and $\sigma_{k} T_{n}=T_{n}\left(\sigma_{k}\right.$ being an element of $\left.G_{n}\right)$.

If $k=i+1$, we note that $\sigma_{k} f(\tilde{x}[i])=\sigma_{i+1} f(\tilde{x}[i])$ is, by definition, $f(\tilde{x}[i+1])+f(\tilde{x}[i])$.

For $k=i$, we have $\sigma_{k} f(\tilde{x}[i])=\sigma_{i}\left(\sigma_{i}-1\right) f(\tilde{x}[i-1])=-f(\tilde{x}[i])$.

For $k=i-1$, we need to show that

$$
\sigma_{k} f(\tilde{x}[i])-f(\tilde{x}[i])-f(\tilde{x}[i-1])=0 .
$$

Now

$$
\begin{aligned}
\sigma_{k} f(\tilde{x}[i])-f(\tilde{x}[i])- & f(\tilde{x}[i-1]) \\
= & \left(\sigma_{i-1}\left(\sigma_{i}-1\right)-\left(\sigma_{i}-1\right)-1\right)\left(\sigma_{i-1}-1\right) f(\tilde{x}[i-2]) \\
= & \left(\sigma_{i-1} \sigma_{i} \sigma_{i-1}-1-\sigma_{i} \sigma_{i-1}-\sigma_{i-1} \sigma_{i}+\sigma_{i-1}+\sigma_{i}\right) f(\tilde{x}[i-2]) \\
= & \left(\sigma_{i} \sigma_{i-1}-\sigma_{i-1}+1\right)\left(\sigma_{i}-1\right) f(\tilde{x}[i-2]) \\
= & \left(\sigma_{i} \sigma_{i-1}-\sigma_{i-1}+1\right)\left(\sigma_{i-2}-1\right) \cdots\left(\sigma_{1}-1\right)\left(\sigma_{i}-1\right) T_{n} x[1] \\
& =0,
\end{aligned}
$$

since $\sigma_{i} T_{n}=T_{n}, \sigma_{i}$ being in $G_{n}$. 
Finally, if $k<i-1$, we have

$$
\begin{aligned}
\sigma_{k} f(\tilde{x}[i]) & =\sigma_{k}\left(\sigma_{i}-1\right) \cdots\left(\sigma_{k+2}-1\right) f(\tilde{x}[k+1]) \\
& =\left(\sigma_{i}-1\right) \cdots\left(\sigma_{k+2}-1\right) \sigma_{k} f(\tilde{x}[k+1]) \\
& =\left(\sigma_{i}-1\right) \cdots\left(\sigma_{k+2}-1\right)(f(\tilde{x}[k])+f(\tilde{x}[k+1])) \\
& =\left(\sigma_{i}-1\right) \cdots\left(\sigma_{k+3}-1\right)(f(\tilde{x}[k+2])) \\
& =\left(\sigma_{i}-1\right) \cdots\left(\sigma_{k+4}-1\right)(f(\tilde{x}[k+3])) \\
& \vdots \\
& =f(\tilde{x}[i]),
\end{aligned}
$$

using the result of the previous case.

To complete the proof of Theorem 1.1 we need to show that $f$ is a bijection. We will achieve this by considering the map induced by $f$ on the indecomposables

$$
Q\left(\tilde{A}^{\otimes n-1}\right)=\frac{I\left(\tilde{A}^{\otimes n-1}\right)}{I\left(\tilde{A}^{\otimes n-1}\right) \cdot I\left(\tilde{A}^{\otimes n-1}\right)} \longrightarrow Q\left(A^{\otimes n-1}\right)=\frac{I\left(A^{\otimes n-1}\right)}{I\left(A^{\otimes n-1}\right) \cdot I\left(A^{\otimes n-1}\right)} .
$$

Here $I(\cdot)$ denotes the augmentation ideal, i.e. the positive degree part of the algebra.

First we need to calculate $T_{n} x[1]$ modulo decomposables, and we shall write $\equiv$ to denote equivalence modulo $I\left(A^{\otimes n-1}\right) \cdot I\left(A^{\otimes n-1}\right)$.

\section{Lemma 2.3.}

$$
T_{n} x[1] \equiv(n-2) ! \sum_{i=1}^{n-1}(n-i) x[i] .
$$

Proof. This will be proved by induction on $n$. For $n=2$, we have $T_{2}=1$ and the result is evident. Now suppose the result holds for $n-1$. Note that

$$
T_{n}=\left(1+\sigma_{n-1}+\sigma_{n-2} \sigma_{n-1}+\cdots+\sigma_{2} \cdots \sigma_{n-2} \sigma_{n-1}\right) T_{n-1},
$$

i.e. the summands of the left-hand factor form a set of coset representatives for $G_{n}$ over $G_{n-1}$. This is easily seen to be the case since, under the natural action of $\Sigma_{n}$ on $\{1,2, \ldots, n\}$, the permutations $\sigma_{j} \cdots \sigma_{n-1}$ each map $n$ differently.

Using the description of the $\Sigma_{n}$ action on primitives given in the proof of Lemma 2.2, we have

$$
\sigma_{j} \sigma_{j+1} \ldots \sigma_{n-1} x[i] \equiv \begin{cases}x[i] & \text { if } j>i+1 \\ x[i]+x[i+1] & \text { if } j=i+1 \\ x[i+1] & \text { if } j \leqslant i\end{cases}
$$


Thus

$$
\begin{aligned}
T_{n} x[1] & \equiv\left(1+\sigma_{n-1}+\sigma_{n-2} \sigma_{n-1}+\cdots+\sigma_{2} \cdots \sigma_{n-2} \sigma_{n-1}\right) T_{n-1} x[1] \\
& \equiv\left(1+\sigma_{n-1}+\sigma_{n-2} \sigma_{n-1}+\cdots+\sigma_{2} \cdots \sigma_{n-2} \sigma_{n-1}\right)(n-3) ! \sum_{i=1}^{n-2}(n-1-i) x[i] \\
& \equiv(n-3) ! \sum_{i=1}^{n-2}(n-1-i)(i x[i+1]+(n-i) x[i]) \\
& \equiv(n-2) ! \sum_{i=1}^{n-1}(n-i) x[i] .
\end{aligned}
$$

Lemma 2.4. The map $f$ is a bijection.

Proof. We begin by using Lemma 2.3 to show that the map induced by $f$ on the indecomposables is an invertible scalar multiple of the identity map $Q\left(\tilde{A}^{\otimes n-1}\right) \rightarrow Q\left(A^{\otimes n-1}\right)$. Note that since $f(\tilde{x}[i])=\left(\sigma_{i}-1\right) f(\tilde{x}[i-1])$ for $i>1$ and $\left(\sigma_{i}-1\right)(x[i-1]) \equiv x[i]$ modulo decomposables, it suffices to prove that $f(\tilde{x}[1]) \equiv \lambda x[1]$ modulo decomposables, for some invertible $\lambda \in R$.

Now,

$$
\left(\sigma_{1}-1\right) x[i] \equiv \begin{cases}-2 x[1] & \text { if } i=1 \\ x[1] & \text { if } i=2 \\ 0 & \text { if } i>2\end{cases}
$$

Hence

$$
\begin{aligned}
f(\tilde{x}[1]) & \equiv\left(\sigma_{1}-1\right) T_{n} x[1] \\
& \equiv\left(\sigma_{1}-1\right)(n-2) ! \sum_{i=1}^{n-1}(n-i) x[i] \\
& \equiv(n-2) !(-2(n-1) x[1]+(n-2) x[1]) \\
& \equiv-n(n-2) ! x[1] .
\end{aligned}
$$

So, the map induced by $f$ is just the identity map multiplied by $-n(n-2) !$, and under the hypotheses of Theorem 1.1 this factor is invertible in the ground ring $R$. In particular then, $f$ induces a bijection on the indecomposables.

It is well known that a morphism of algebras that induces a surjection on the indecomposables is a surjection itself, and in the case where $A$ is of finite type, surjectivity implies bijectivity, completing the proof of the lemma in this case.

In the non-finite type situation we must work harder to deduce injectivity. Suppose $y \in$ $\tilde{A}^{\otimes n-1}$ is in the kernel of $f$. We assert that $y$ lies in some subalgebra of $\tilde{A}^{\otimes n-1}$, generated by a finite number of primitive elements $\tilde{x}[i]$, which is mapped by $f$ to the corresponding subalgebra of $A^{\otimes n-1}$ (generated by the elements $x[i]$ ). These subalgebras are isomorphic 
and of finite type, so the above argument applies to show that the restriction of $f$ is bijective and thus $y$ must be 0 .

To prove the existence of such a subalgebra, we construct a list of its generators. Since $\tilde{A}^{\otimes n-1}$ is generated by primitives, we can choose a finite number of such elements such that $y$ lies in the subalgebra they generate. We begin our list with these elements. In general this will not suffice: if $\tilde{x}[i]$ is primitive, $f(\tilde{x}[i])+n(n-2) ! x[i]$ may well not be in the subalgebra of $A^{\otimes n-1}$ generated by the corresponding elements. Nevertheless, it will be decomposable and will lie in the subalgebra generated by a certain finite collection of $x[i]$ 's for $\tilde{x}$ primitive, of lower degree than the original element $\tilde{x}[i]$. We add the corresponding $\tilde{x}[i]$ 's to our list. Repeating this for all of the initial list gives us the second stage of the list. Carrying out the same process for all of the second-stage elements yields the third stage of the list and so on. The fact that the elements added at each stage have strictly lower degree than the maximum degree occurring in the previous stage, means there can only be finitely many stages to the process. Since we only add finitely many elements at each stage, this guarantees that the process will end with a finite list.

This completes the proof of Theorem 1.1.

Acknowledgements. M.D.C. was supported by a grant from the Max-Planck-Institut für Mathematik and thanks this organization for its support and hospitality and for paying for a visit by S.W. We both thank Ran Levi for his comments on Molien's Theorem and the referee for advice on improving the paper.

\section{References}

1. J. F. AdAms, Lectures on generalised cohomology, Springer Lecture Notes in Mathematics, vol. 99, pp. 1-138 (Springer, 1969).

2. D. J. Benson, Polynomial invariants of finite groups, London Mathematical Society Lecture Note Series, vol. 190 (1993).

3. M. D. Crossley and S. Whitehouse, On conjugation invariants in the dual Steenrod algebra, Proc. Am. Math. Soc. 128 (2000), 2809-2818.

4. R. Kane, The homology of Hopf spaces (North Holland, Amsterdam, 1988).

5. J. Milnor And J. Moore, On the structure of Hopf algebras, Ann. Math. 81 (1965), $211-264$.

6. A. Robinson and S. Whitehouse, $\Gamma$-homology of commutative rings and of $E_{\infty}$-ring spectra, Warwick preprint 76/1995.

7. L. Smith, Polynomial invariants of finite groups (A. K. Peters Ltd, Wellesley, MA, 1995).

8. S. Whitehouse, Symmetric group actions on tensor products of Hopf algebroids, Université d'Artois preprint 99-5. 